\title{
La prueba de claudicación por rascado para diagnosticar lesiones de nervio periférico
}

\author{
G. González-Trevizo(1), J. BARbour ${ }^{(2)}$, S. E. MACKinnon ${ }^{(3)}$, \\ F. García De LuCas $^{(4)}$, M. Cuadros Romero ${ }^{(5)}$ \\ (1) Fellow de Nervio Periférico del Hospital Barnes-Jewish de la Washington University, St. Louis Mo, \\ USA \\ (2) Fellow de Cirugia de Mano del Hospital Barnes-Jewish de la Washington University, St. Louis Mo, \\ USA \\ (3) Jefe de Servicio de Cirugía Plástica del Hospital Barnes-Jewish de la Washington University, St. \\ Louis Mo, USA. División of Plastic and Reconstructive Surgery, Washington University School of \\ MEDICINE, ST. LOUIS, Mo, USA \\ (4) Director Médico Nacional Fremap. Unidad de Miembro Superior Hospital Fremap. Centro de Preven- \\ CIÓN Y REHABILITACIÓN. MAJADAHONDA. MADRID \\ (5) Jefe de Sección del Servicio Cot. Hospital Clínico Universitario Virgen de la Victoria. Málaga. \\ ESPAÑA
}

Correspondencia:

Dr. Gilberto González-Trevizo

Division of Plastic and Reconstructive Surgery

Washington University School of Medicine

660 South Euclid Avenue, Campus Box 8238

St. Louis, MO 63110, USA

Teléfono: +1(314)562-0882

e-mail: gontregil@gmail.com

Introducción: La prueba de claudicación por rascado es una prueba provocativa recientemente descrita para el diagnóstico de compresiones nerviosas periféricas

Método: El examinador se coloca sentado frente al paciente, primero sin provocar estimulo previo, y le intenta rotar internamente los hombros a lo que el paciente se resiste. Acto seguido se rasca la piel del paciente suavemente sobre el área de la compresión nerviosa, mientras el paciente se resiste a una rotación interna bilateral de los hombros con los codos flexionados, si el paciente tiene alodinia debido a una neuropatía compresiva, se obtiene una momentánea pérdida de la resistencia muscular.

Discusión: Los resultados obtenidos son en base a los sitios de compresión del nervio periférico, y son objetivos porque el examinador percibe el colapso del paciente
Background: The Scratch Collapse Test is a recently described provocative test for diagnosis of peripheral nerve compression.

Method: The examiner is placed facing the patient, first without cause prior stimulus, is trying to internally rotate the shoulders and the patient resists, scratches immediately afterwards the patient's skin gently on the area of nerve compression, while the patient resists internal rotation of the shoulders bilaterally with elbows bent, if the patient has allodynia due to a compressive neuropathy, you get a momentary loss of muscular resistance.

Discussion: The results are based on the sites of peripheral nerve compression are objective because the examiner feels the collapse of the patient Conclusion: This test can be reproduced easily and does not require a big learning curve, we are working to demonstrate the exact physiological 
Conclusión: Esta prueba puede ser reproducida con facilidad una vez que se pase la curva de aprendizaje. Se esta trabajando en demostrar exactamente el mecanismo fisiológico de como funciona, y también se están analizando otras partes del cuerpo en donde se puede obtener el colapso.

Palabras clave: prueba de claudicación por rascado, lesiones de nervio periférico. mechanism of how it works and are also looking at other parts of the body where you can obtain the collapse.

Key words: scratch-collapse test, Peripheral nerve injuries.

\section{INTRODUCCIÓN}

$\tau$ as neuropatías compresivas son comunes tanto en las extremidades superiores como en las inferiores. El cuadro clínico puede involucrar síntomas motores o sensitivos en la distribución del nervio comprometido. $\mathrm{Pa}-$ ra el diagnóstico de las neuropatías compresivas el médico se apoya en pruebas de provocadoras como el signo de Tinel, Phalen y Durkan ${ }^{1-3}$, que demuestran la compresión, y en métodos auxiliares como son la ecografía, resonancia magnética y estudio electrodiagnóstico. Estos últimos mucha de las veces no son exactos ${ }^{4}$. Los datos de sensibilidad reportados varían de $49 \%$ a $84 \%$ en la evaluación de síndrome de túnel del carpo ${ }^{5}$. Es por eso que en ocasiones el realizar un diagnóstico adecuado de lesión de nervio periférico o neuropatía compresiva representa un reto para el clínico ${ }^{6,7}$.

Todos sabemos que los estudios auxiliares de diagnóstico se tienen que usar, como su nombre lo indica, para apoyar o descartar un diagnóstico que ya se ha pensado, y no para encontrar algo de lo cual no sabemos que es. De esta manera el examen clínico y las pruebas diagnósticas deben tener una buena sensibilidad y especificidad, que puedan realizarse con facilidad, ser confiables y reproducibles ${ }^{8}$.

La mayoría de las pruebas clínicas se basan en que el paciente nos informe lo que siente, lo que las convierte en pruebas subjetivas. Las lesiones de nervio periférico incluyendo las neuropatías compresivas, pueden causar dolor neuropático caracterizado por hiperalgesia (respuesta exage- rada a un estimulo doloroso) y alodinia (respuesta dolorosa de un estimulo no doloroso) ${ }^{9-11}$. Clínicamente, y en modelos en ratas, estos ocurren con estimulación cutánea dentro y fuera del territorio del nervio comprometido. Se ha observado que estímulos cutáneos dolorosos causan un período de inhibición en la actividad muscular voluntaria tónica en humanos. Este periodo de silencio eléctrico se ha denominado periodo $\mathrm{cu}$ táneo silencioso ${ }^{12,13}$. Aunque el mecanismo aún no esta bien entendido, se acepta generalmente que un reflejo espinal inhibitorio puede jugar un rol de protección al facilitar la retirada de la extremidad de un potencial estimulo doloroso ${ }^{14,15}$.

\section{MÉTODO}

La prueba de claudicación por rascado introducida por la Dra. Susan E. Mackinnon para el diagnóstico de los síndromes de túnel del carpo y cubital ${ }^{8}$, consiste en que el examinador se coloca sentado frente al paciente, que también esta sentado pero sin apoyar su espalda en el respaldo para que no interfiera el bloqueo de los músculos escapulares (aunque resultados similares se han obtenido con el paciente de pie) (Figura 1). El paciente coloca los hombros en posición neutra, los codos flexionados a 90 grados, antebrazos en posición neutra, y las muñecas en extensión. El examinador empuja los brazos del paciente hacia adentro aplicando la presión sobre las muñecas y no sobre las manos, y pide al paciente que resista a esta fuerza. Acto seguido se rasca suavemente la piel del paciente sobre el área de la compre- 


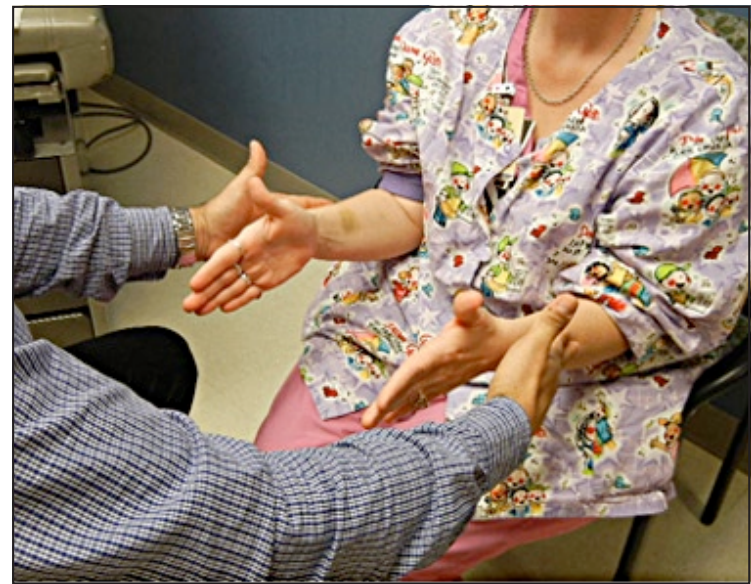

Figura 1. El examinador sentado frente al paciente prueba la fuerza del paciente para establecer una basal, antes de realizar el rascado.

sión nerviosa mientras el paciente se resiste a una rotación bilateral interna de los hombros (Figura 2), Si el paciente tiene una neuropatía compresiva, se obtiene una momentánea pérdida de la resistencia muscular (Figura 3). La ventaja es que esta prueba no depende de los datos subjetivos que pueda aportar el paciente y si depende de los datos objetivos que el examinador pueda encontrar.

Se agrega como complemento de esta prueba el cloruro de etilo (Figura 4), sustancia utilizada para anestesiar de forma temporal la piel cuando se van a colocar catéteres intravenosos o en tratamientos de síndromes miofasciales. Existen lugares en donde el acceso al cloruro de etilo no es fácil, por lo que valdría la pena valorar si se puede obtener la misma respuesta con xilocaina

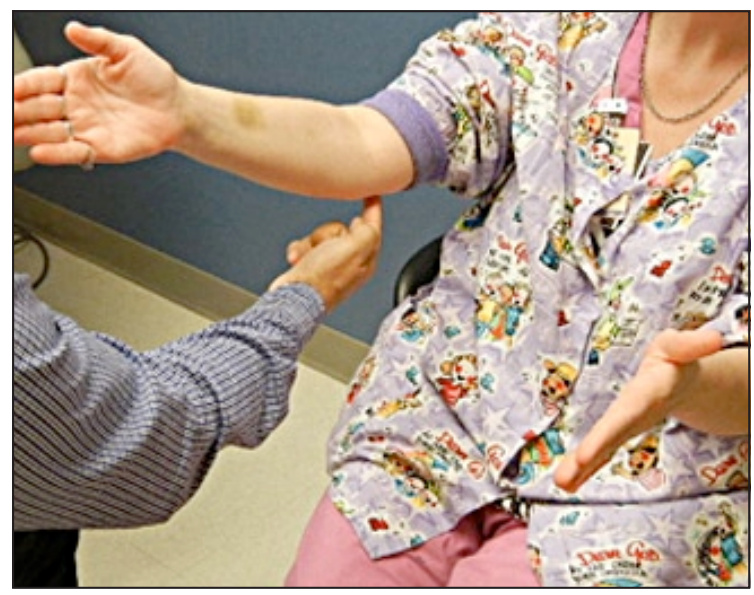

Figura 2. El examinador rasca sobre el nervio a explorar.

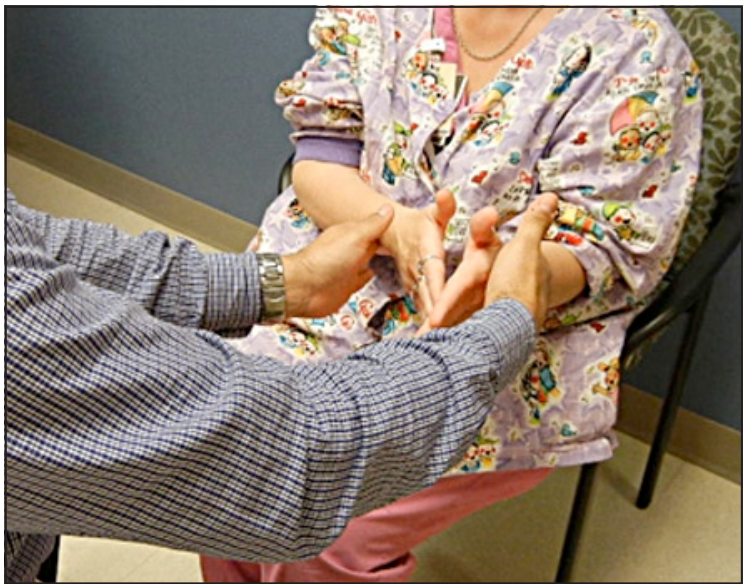

Figura 3. Se repite la aplicación de la fuerza al paciente y si existe una compresión nerviosa, este va a «colapsar».

en spray. La razón de aplicar cloruro de etilo, es para corroborar la prueba y localizar nuevos sitios de colapso.

Primero se realiza la prueba de manera convencional. Una vez que se encontraron los puntos de colapso primario y secundarios, se procede a anestesiar con cloruro de etilo la piel en la zona principal de colapso. Después se repite el examen, cuidando de utilizar diferente dedo estimulador para no propagar el anestésico.

Una vez anestesiada la zona esta ya no colapsa puesto que ya no se propaga el estimulo cutáneo (Figura 5). Lo que se encuentra a continuación es que los puntos de colapso secundarios ahora

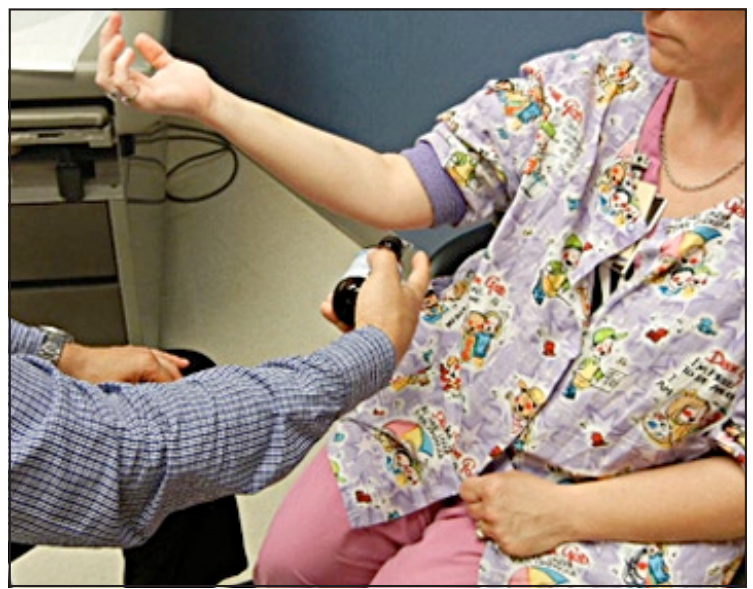

Figura 4. Cuando se quiere corroborar la prueba o cuando se quieren buscar sitios secundarios o subclínicos, se aplica cloruro de etilo en el sitio donde el rascado dio positivo previamente. 


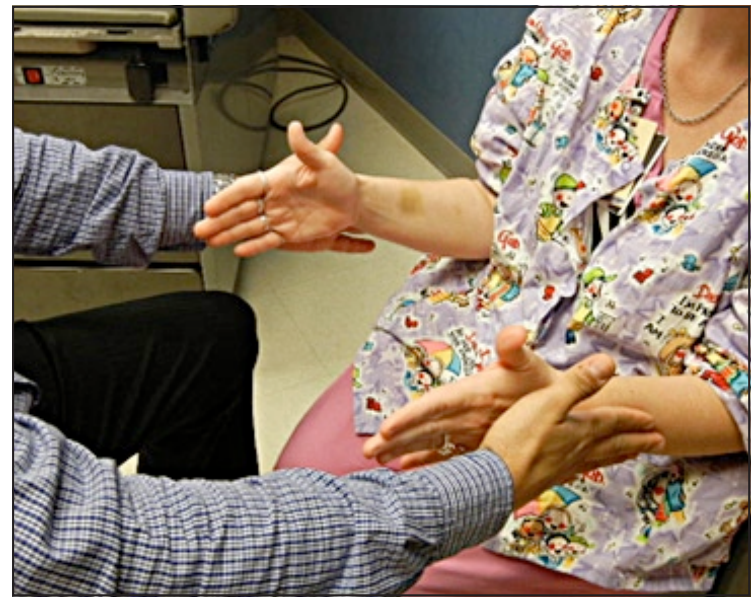

Figura 5. Después de haber aplicado el cloruro de etilo, se rasca nuevamente sobre la zona previamente examinada y el examen ahora será negativo, eso significa que el paciente no colapsará, puesto que ya no existe estímulo cutáneo.

colapsan con mayor facilidad y muchas veces aparecen puntos en zonas donde no colapsaban antes (Figuras 6 y 7).

\section{DISCUSIÓN}

En la descripción original de la prueba ${ }^{8}$ se recomendaba para diagnostico del túnel del carpo y túnel cubital, pero ahora sabemos que esta prueba puede ser utilizada para cualquier neuropatía compresiva y en lesiones nerviosas como evaluación pre y postquirúrgica, así como factor

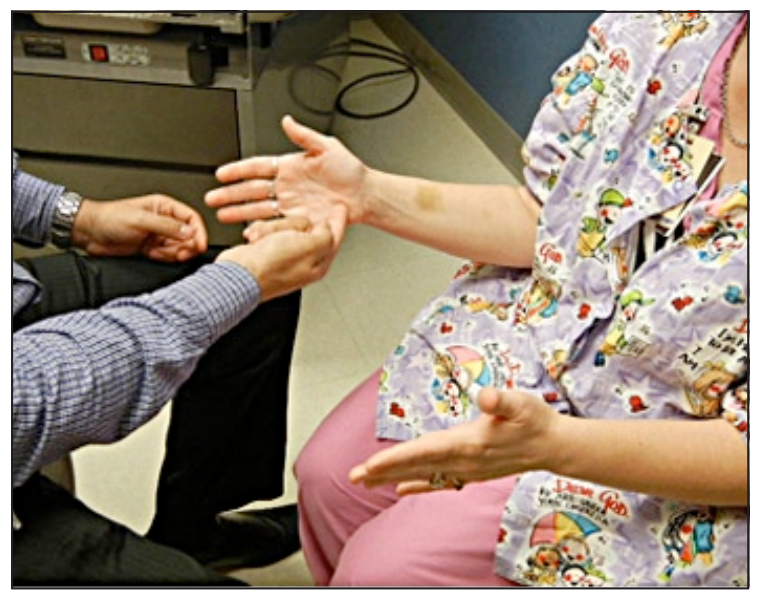

Figura 6. Sitios «subclínicos» que previamente eran negativos, se estimulan nuevamente con un rascado (como el canal de Guyon que aquí se presenta). pronóstico. Sugerimos que cuando al paciente se le haga el estimulo de rascado sobre la zona a examinar, concomitantemente no se estimule ninguna otra zona, puesto que la prueba puede arrojar resultados cruzados. Así mismo dependiendo de la localización anatómica del nervio a examinar la aplicación del estimulo puede incluir: rascado, presión suave o soplar (puesto que se estimulan más fibras nerviosas, principalmente sensitivas). Actualmente se esta trabajando en transpolar el uso de la resistencia a la rotación interna en las extremidades superiores al uso de la resistencia a la inversión en los tobillos, de aplicación en situaciones en que el paciente no pueda resistir la rotación interna del hombro por motivos diversos, que pueden incluir fracturas recientes de extremidad superior o lesiones completas de plexo braquial.

Tradicionalmente se utiliza para diagnosticar las neuropatías compresivas el examen clínico que incluyen pruebas como Tinel, Phalen y Durkan, junto con los estudios de electrodiagnóstico ${ }^{16}$. Otras maniobras de provocación pueden utilizarse para complementar el armamento diagnóstico ${ }^{17}$. En el estudio realizado por Mackinnon y Cheng ${ }^{8}$ se demostró la utilidad y fiabilidad de la prueba de colapso por rascado en pacientes con electromiografía positivas, y se comprobó que esta prueba tiene una mayor sensibilidad $(\mathrm{p}<.001)$ que la prueba de Tinel. La exactitud fue de $82 \%$ para diagnosticar túnel del carpo y de $89 \%$ para el túnel cubital. Existe una curva de aprendizaje para esta prueba en general

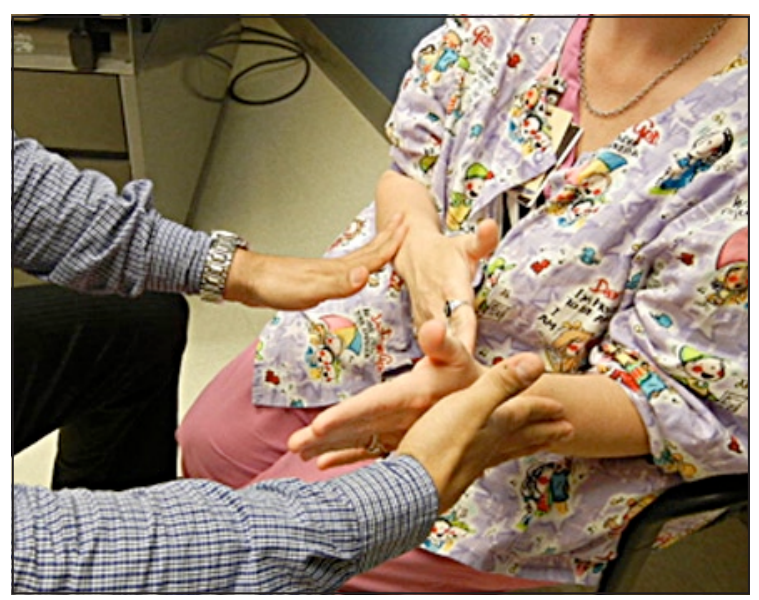

Figura 7. Estos sitios previamente negativos, ahora serán positivos. 
y también para el examinador que la comienza a utilizar, puesto que se deben controlar diversas variables como son la posición del paciente, la cantidad de la fuerza cuando se intenta provocar el colapso, así como el tipo de estímulo y la localización anatómica exacta donde este se aplica.

Otra ventaja importante a mencionar es el hecho de que como es una prueba nueva es más difícil para el paciente, que busca una ganancia secundaria, que pueda fingir la misma.

Puede realizarse varias veces por el mismo examinador, o por examinadores diferentes, sin que esto cambie los resultados obtenidos. $\mathrm{Mu}-$ chas veces, cuando los sitios de compresión son en diferentes lugares de la trayectoria del mismo nervio, se debe de priorizar y estatificar la magnitud del colapso. Esto quiere decir que siempre se encuentra un punto de mayor colapso y puntos de menor colapso, y en donde colapsa con mayor facilidad es el sitio de mayor compresión.

Se piensa que la razón del colapso se debe a la mediación principalmente de fibras de conducción lenta $\mathrm{A}-\delta \mathrm{y}$, probablemente, a un reflejo espinal protector inhibitorio ${ }^{18}$. Ya se habían elaborado trabajos en donde por medio de electromiografía se registraba la respuesta eléctrica, del arco reflejo de retirar una extremidad después de un estimulo doloroso ${ }^{19}$.

Como se mencionó anteriormente el mecanismo exacto de como funciona esta prueba se desconoce. Creemos que representa una manifestación física del periodo cutáneo silencioso (fenómeno electromiográfico que se observa como respuesta a un estímulo).

Una pausa breve de la contracción muscular voluntaria se demuestra tras la estimulación de un nervio cutáneo. Se piensa que cuando se estimulan los nervo-nervorum en el sitio de la neuritis, una inhibición central ipsilateral tran- sitoria se activa, lo que se encuentra con mayor claridad en donde existe el mayor daño o la mayor compresión.

Esta prueba también sirve cuando estamos buscando sitios de regeneración nerviosa, secundario a lesiones y electromiograficamente no se encuentran aun datos de reinervacion ${ }^{20}$.

\section{CONCLUSIÓN}

La prueba del colapso por rascado, es un examen fácil de aprender, con una curva de aprendizaje, sencillo de realizar, con molestias mínimas para el paciente, gratuito y que ofrece muchas ventajas sobre las pruebas convencionales. Tiene una mejor sensibilidad y especificidad sobre otras pruebas, localiza el punto exacto de lesión sobre el trayecto del nervio, prioriza los sitios de compresión, es autocomprobable con cloruro de etilo que nos ayuda a localizar otros sitios de menor compresión. Sirve para realizar un diagnóstico y para el seguimiento postquirúrgico.

En casos de lesiones de nervio periférico, puede identificar sitios de reinervación inclusive antes de que la electromiografía convencional los detecte. Por lo tanto consideramos importante su difusión en la comunidad médica para que de esta forma sus ventajas puedan ser reproducidas, y también para que cuantos más médicos la utilicen se puedan realizar correcciones y enmiendas a la misma. El futuro de esta prueba es demostrar de forma exacta el mecanismo de como funciona fisiológicamente hablando, así como encontrar si realmente se puede utilizar en los tobillos para que nos pueda servir en pacientes en los cuales no se les puede pedir que resistan una rotación interna de hombros. 


\section{BIBLIOGRAFÍA}

1. Katz JN, Larson MG, Sabra A Krarup C, et al. The carpal tunnel syndrome: diagnostic utility of the history and physical examination findings. Ann Intern Med, 1990; 112: 321-7.

2. Durkan JA. A new diagnostic test for carpal tunnel syndrome. J Bone Joint Surg Am, 1991; 73: 535-8.

3. Williams TM, Mackinnon SE, Novak CB. Verification of the pressure provocative test in carpal tunnel syndrome. Ann Plast Surg, 1992; 29: 8-11.

4. LaJoie AS, McCabe SJ, Thomas B. Determining the sensitivity and specificity of common diagnostic tests for carpal tunnel syndrome using latent class analysis. Plast Reconstr Surg, 2005; 116: 502-7.

5. American Academy of Neurology, American Association of Electrodiagnostic Medicine, and American Academy of Physical Medicine and Rehabilitation. Practice parameter for electrodiagnostic studies in carpal tunnel syndrome. Neurology, 1993; 43: 2404-5.

6. Massy-Westropp N, Grimmer $\mathrm{K}$, Bain G. A systematic review of the clinical diagnostic tests for carpal tunnel syndrome. J Hand Surg Am, 2000; 25: 120-7.

7. MacDermid JC, Wessel J. Clinical diagnosis of carpal tunnel syndrome: a systematic review. J Hand Ther, 2004; 17: 30919.

8. Cheng CJ, Mackinnon-Patterson B, Beck JL, Mackinnon SE. Scratch collapse test for evaluation of carpal and cubital tunnel syndrome. J Hand Surg Am, 2008; 33: 1518-24.

9. Sheen K, Chung JM. Signs of neuropathic pain depend on signals from injured nerve fibers in a rat model. Brain Res, 1993; 610: 62-8.

10. Tal M, Eliav E. Abnormal discharge originates at the site of nerve injury in experimental constriction neuropathy $(\mathrm{CCl})$ in the rat. Pain, 1996; 64: 511-8.

11. Sotgiu ML, Biella G. Contribution of central sensitization to the pain-related abnormal activity in neuropathic rats. Somatosens Mot Res, 2000; 17: 32-8.

12. Uncini A, Kujirai T, Gluck B. Silent period induced by cutaneous stimulation. Electroencephalogr Clin Neurophysiol, 1991; 81: 344-52.

13. Logigian EL, Plotkin GM, Shefner JM. The cutaneous silent period is mediated by spinal inhibitory reflex. Muscle Nerve, 1999; 22: 467-72.

14. Leis AA, Stokic DS, Fuhr P, et al. Nociceptive fingertip stimulation inhibits synergistic mo- toneuron pools in the human upper limb. Neurology, 2000; 55: 1305-9.

15. Kofler M. Functional organization of exteroceptive inhibition following nociceptive electrical fingertip stimulation in humans. Clin Neurophysiol, 2003; 114: 973-80.

16. Rempel D, Evanoff B, Amadio $\mathrm{PC}$, deKrom M, et al. Consensus criteria for the classification of carpal tunnel syndrome in epidemiologic studies. Am J Public Health, 1998; 88: 1447-51.

17. Novak CB, Lee GW, Mackinnon SE. Provocative testing for cubital tunnel syndrome. $J$ Hand Surg Am, 1994; 19: 817-20.

18. Serrao M, Parisi L, Pierelli F. Cutaneous afferents mediating the cutaneous silent period in the upper limbs: evidences for a role of low-threshold sensory fibres. Clin Neurophys, 2001; 112: 2007-14.

19. Floeter MK, Gerloff C, Kouri J. Cutaneous withdrawal reflexes of the upper extremity. Muscle Nerve, 1998; 21: 591-8.

20. Brown JM, Mokhtee D, Evangelista MS, Mackinnon SE. Scratch collapse test localizes Osborne's band as the point of maximal nerve compression in cubital tunnel syndrome. Hand, 2010: 5: 141-7. 